\title{
FDG uptake observed around the lumbar spinous process: relevance to Baastrup disease.
}

\section{AUTHOR(S):}

Nishimatsu, Kayo; Nakamoto, Yuji; Ishimori, Takayoshi; Togashi, Kaori

\section{CITATION:}

Nishimatsu, Kayo ... [et al]. FDG uptake observed around the lumbar spinous process: relevance to Baastrup disease.. Annals of nuclear medicine 2015, 29(9): 766-771

\section{ISSUE DATE:}

2015-11

URL:

http://hdl.handle.net/2433/203017

\section{RIGHT:}

The final publication is available at Springer via http://dx.doi.org/10.1007/s12149-015-1003-5.; The full-text file will be made open to the public on 14 July 2016 in accordance with publisher's 'Terms and Conditions for Self-Archiving'.; この 論文は出版社版でありません。引用の際には出版社版をご確認ご利用ください。; This is not the published version. Please cite only the published version. 


\section{FDG uptake observed around the lumbar spinous process: relevance to Baastrup disease}

Short title: Lumbar spinous process FDG uptake

Kayo Nishimatsu MD *, Yuji Nakamoto MD PhD*, Takayoshi Ishimori MD PhD*, Kaori Togashi $\mathrm{MD} \mathrm{PhD*}$

*Department of Diagnostic Imaging and Nuclear Medicine, Graduate School of Medicine, Kyoto

University

Corresponding Author: Yuji Nakamoto, MD, PhD

Department of Diagnostic Imaging and Nuclear Medicine,

Graduate School of Medicine, Kyoto University

Tel: +81-75-751-3762

Fax: +81-75-771-9709

E-mail: ynakamo1@kuhp.kyoto-u.ac.jp

Type of article: original article 
Lumbar spinous process FDG uptake

\begin{abstract}
Objective:

In positron emission tomography (PET) with F-18-fluorodeoxyglucose (FDG), non-tumorous focal uptake is often observed around the lumbar spinous processes (LSPs). Close approximation of LSPs with sclerosis is often seen, which is called Baastrup disease. The aim of this study was to characterize this finding in terms of location and subjects' age and investigate the relation between PET and CT findings.
\end{abstract}

\title{
Methods:
}

The PET/CT scans of 40 patients each in the fifth, sixth, seventh, eighth, and ninth decades were screened for FDG uptake around the LSPs from L1-2 through L5-S1. Patients with metastasis to the lumbar spine or recent chemotherapy or rheumatoid arthritis-related disease were excluded. Focal uptake greater than blood pool activity was considered positive. Positive uptake was compared among the ages and locations. We also evaluated the relationship between FDG uptake and CT morphology.

\section{Results:}

Overall, focal uptake was observed in 122 LSPs in 71 patients. At least one positive uptake was seen in $9,21,15,12$, and 14 of 40 patients $(16,30,30,20$, and 26 of 200 regions) in each age group of 40's through 80 's, respectively ( $\mathrm{p}=0.12$ ). As for the 
Lumbar spinous process FDG uptake

location, uptake around L1-2, L2-3, L3-4, L4-5, and L5-S1 was observed in 19, 22, 39,

35 , and 7 regions, respectively $(\mathrm{p}<0.01)$. There was no statistically significant difference in PET positivity among the five age groups, but positive uptake was predominantly seen in L3-4. Degeneration on CT was apparent in 58, 74, 108, 123, and 144 regions in each age group, respectively ( $\mathrm{p}<0.01)$, and in $38,79,131,151$, and 108 regions in each location, respectively $(\mathrm{p}<0.01)$. The PET positive ratio was higher in CT positive group than in CT negative group ( $14 \%$ vs. $10 \%, \mathrm{p}<0.05)$, but there was no significant difference of quantitative values $(\mathrm{p}=0.10)$. Of 42 regions in 27 patients who had serial PET/CT scans that were initially PET-positive, 35 regions (83\%) turned negative on a later PET-scan.

\section{Conclusions:}

Focal uptake around the LSPs was commonly seen in the mid-lumbar vertebrae, independent of age, and was not always correlated with morphological changes. This uptake should not be assumed to represent osseous metastasis.

Key Words: Positron-emission tomography; Computed Tomography; Spine; Lumbar spinous process; Baastrup disease 
Lumbar spinous process FDG uptake

\section{Introduction}

Positron emission tomography (PET) using F-18-fluorodeoxyglucose (FDG) is a useful modality to detect metabolically active lesions, and has been widely accepted as being especially helpful in staging, restaging, and monitoring therapeutic response in many malignant diseases [1]. However, it is well known that FDG is not a tumor-specific agent, and accumulates in active inflammatory foci as well. Therefore, when interpreting PET images, we need to consider whether elevated FDG uptake is representing a malignant tumor or a benign process.

There are some tissues in which FDG accumulates physiologically [2], such as brain, myocardium, liver, and bowel. Benign FDG avidity has been reported in degenerative arthritis and inflammatory diseases [3-5]. FDG uptake in degenerative spinal changes, such as osteophytes, disk disease, and facet joint disease, is quite common [3,4]. It is also observed around the lumbar spinous processes (LSPs). Baastrup disease, which is also known as a kissing spine, is characterized by the close approximation and contact of adjacent spinous processes with sclerosis [6-11]. The morphological appearance and features on computed tomography (CT) or magnetic resonance (MR) images have been demonstrated [11-16]. The characteristics and incidence of this entity on FDG-PET have not been fully investigated, although there 
Lumbar spinous process FDG uptake

are some case reports demonstrating localized FDG uptake between two adjacent

spinous processes with Baastrup disease [17-19].

The purpose of this study was to investigate the characteristics of FDG uptake around the LSPs, which may be due to Baastrup disease, in terms of location, CT findings, patient age, and time course.

\section{Materials and Methods}

Patients

To ensure an equal age spread, patients were stratified into five age categories (40s, 50s, 60s, 70s, and 80s). For each age category, 20 consecutive male patients and 20 female patients, who underwent whole body FDG-PET/CT scan from May 2009 to October 2009 in our institute, were selected for analysis; a total of 200 patients were analyzed. Patients who had bone metastasis in the lumbar spine, rheumatoid arthritis-related disease, such as polymyalgia rheumatica or seronegative spondyloarthropathy, or who had received chemotherapy or radiotherapy to the lumbar vertebrae within 3 months before the examination were excluded. For all eligible patients, we confirmed the absence of bone metastasis for a follow-up period ending October 2013 by examining medical records, CT, or MRI findings after their PET/CT 
Lumbar spinous process FDG uptake

examinations. For patients who had positive PET findings and underwent another

PET/CT scan during the follow-up period without chemo/hormonal therapy, we

evaluated the time-dependent change of PET and CT findings. This retrospective study

was approved by the institutional review board and ethics committee of our institute,

and written informed consent was obtained from each patient for their data access.

PET/CT scanning

Patients fasted for at least $4 \mathrm{~h}$ before the examination, and their plasma glucose level was measured just before the administration of F-18-FDG (3.7 MBq/kg of body weight). There were no patients with a plasma glucose level greater than $200 \mathrm{mg} / \mathrm{dL}$ $(11.1 \mathrm{mmol} / \mathrm{L})$ in this population. Approximately $1 \mathrm{~h}$ after injection, PET/CT images were acquired with a PET/CT scanner (Discovery ST Elite; GE Healthcare, Waukesha Wisconsin, USA) that integrated a bismuth germinate crystal PET system with a 16-slice multi-detector-row CT scanner. CT scanning was performed immediately before PET emission scanning using the following settings: $120 \mathrm{kVp}$ tube voltage, 20-100mA tube current (automatic setting), $0.6 \mathrm{~s}$ per tube rotation, pitch 1.75, and 3.75 mm section thickness. CT images were reconstructed by filtered back projection into $512 \times 512$ pixel images. PET emission scanning was performed with an acquisition time 
Lumbar spinous process FDG uptake

of 2-3 min per bed position. The data were reconstructed into $128 \times 128$ pixel images

with a three-dimensional iterative reconstruction algorithm using CT-based attenuation

correction.

Image Analysis

PET/CT images were evaluated by two physicians, each of whom was a

board-certified radiologist and nuclear medicine physician, using consensus in cases of disagreement. The PET/CT data of all 200 patients were evaluated on a workstation (Advantage workstation version 4.4; GE Healthcare, Waukesha WI, USA) with CT multiplanar reconstruction images of $3.27 \mathrm{~mm}$ thickness. Two CT criteria were assessed: 1) close approximation and contact between apposed spinous processes, 2) sclerosis of the superior and inferior portions of adjacent processes. The presence of both was scored as positive on CT [7-11]. PET images were evaluated in terms of focal increased FDG uptake around the LSPs. The uptake in a region was considered positive on PET when it was localized and greater than the blood pool activity, defined as the activity in the aortic arch. The maximum standardized uptake value (SUVmax) was calculated in PET-positive cases. Positivity was compared among the age decades (fifth through ninth) and locations (L1-2, L2-3, L3-4, L4-5, and L5-S1). Further, we evaluated 
Lumbar spinous process FDG uptake

the relationship between uptake on PET and morphological changes on CT. If a patient had serial PET/CT scans, temporal changes in the findings were evaluated. Statistical analysis was performed using the chi-squared test and Mann-Whitney $U$ test. A $P$ value $<0.05$ was considered statistically significant.

\section{Results}

In 1000 regions in 200 patients, positive FDG uptake around the LSP was observed in 122 regions in 71 patients (63 regions in 35 males and 59 regions in 36 females). The SUVmax was $0.9-3.1$, with a mean of 1.53 . There was no statistically significant difference in overall PET positivity between males and females. Degenerative changes on CT images were observed in 507 regions in 170 patients (227 regions in 81 males and 280 regions in 89 females). No statistically significant gender difference was found in CT positivity. A representative case is shown in Figure 1.

Figure 2 (patients) and Table 1 (regions) show the distribution of positive uptake foci by decade. There was no statistically significant difference in PET positivity among these five age groups (Chi-square test, $P=0.12$ ). Conversely, the frequency of degenerative changes on CT increased with age (Chi-square test, $P<0.01$ ) (Table 1).

Table 2 displays PET-positive uptake according to location in the lumbar spine. 
Lumbar spinous process FDG uptake

Positive uptake on PET was predominantly seen in the mid-lumbar region, especially in

L3-4, and rarely seen in L5-S1. There was statistically significant difference in PET

positivity among location (Chi-square test, $P<0.01)$. While degenerative changes on

CT tended to be observed in the mid- to lower-lumbar spine. There was statistically

significant difference in CT positivity among location (Chi-square test, $P<0.01$ ).

Increased FDG uptake was identified in 72 of 507 regions (14\%) with

degenerative change and in 50 of 493 regions (10\%) without degenerative change on

CT (Chi-square test, $P<0.05)$. In addition, regions with morphological changes on CT

tended to show higher uptake, without statistical significance (Mann-Whitney $U$ test,

$P=0.10)($ Table 3).

Of 71 patients with positive PET findings, 27 underwent another PET/CT scan

and during the follow-up period. These patients did not undergo chemotherapy,

hormonal therapy, intensive steroid or immunosuppressant treatment. The second scans

were performed 3-57 months (mean 16.3 months) after the initial scan. The time

courses of PET and CT findings are demonstrated in Figure 3. Thirty-five of 42 regions

$(83 \%)$ with positive FDG uptake on the first scan turned negative on the second scan,

whereas all 62 regions with positive CT findings on the first scan remained positive on

the second scan. In 17 regions with FDG uptake, but no morphological changes on CT, 
Lumbar spinous process FDG uptake

on the first scan, degenerative changes appeared on the second scan in only four regions

(24\%). In 135 regions in these 27 patients, CT findings changed from negative to

positive in 10 regions, while did not change in the remaining 125 regions. There was no significant difference of intervals between the changed group and the unchanged group

(Mann-Whitney $U$ test, $P=0.21$ ).

\section{Discussion}

Uptake around the LSP was often seen in the mid-lumbar spine independent of age, while morphological degenerative changes on CT predominated in the lower lumbar spine in elderly persons. In some regions, the uptake corresponded to degeneration observed in Baastrup disease. However, there were some regions where the uptake was seen without any degenerative changes on CT. Therefore, it is considered the uptake around the LSP is not always correlated with the morphological changes; Baastrup disease.

Baastrup disease is characterized by the close approximation and contact of adjacent spinous processes with associated osteosclerosis on CT, and bursitis on MRI. It has been reported that repetitive strain on the interspinous ligaments leads to their degeneration and collapse, causing traction enthesopathy and formation of interspinous 
Lumbar spinous process FDG uptake

bursae $[5,7-9,12]$, with osseous proliferation and eburnation of the spinous processes

$[3,7,9]$. In our subjects, a statistically significant difference was found in positive uptake ratios, dependent on the presence or absence of morphological changes on CT (Table 3).

This suggests that focal FDG uptake around the LSP may be related to degenerative changes. Focal FDG uptake was seen in about $10 \%$ of cases when degenerative change was not observed on CT. In these cases, uptake might reflect inflammation or rupture of interspinous ligament, or bursitis, as reported by MRI studies [11-16].

It is reported that Baastrup disease occurs with higher frequency in older patients [9-11]. Our findings are consistent with this. In addition, Aliyev et al. reported that intensity of FDG uptake (SUVmax) in the spinous processes generally increases with age without statistically significant difference [20]. In our population, focal FDG uptake around the LSP was predominantly observed in middle-aged patients (50s-60s), though not to a statistically significant degree, which is also considered similar with the previous reports.

The peak age for PET positivity was somewhat earlier than that for CT positivity, which may be posited to be due to FDG accumulation, reflecting an earlier stage of degenerative change of the spinous processes or interspinous ligaments, and/or interspinous bursitis not visible on CT. Degenerative change on CT was commonly seen 
Lumbar spinous process FDG uptake

in the lower lumbar spine, but FDG uptake was predominantly seen in the mid-lumbar spine, and was an unusual finding at L5-S1. Focal FDG uptake around the LSP and degenerative changes on CT were similar in distribution except at L5-S1. Owing to lordosis of the lumbar spine, the spinous processes of L5 is originally and commonly closed to that of S1. Moreover, the shape of lumbar vertebrae is different from that of sacral vertebrae. Each lumbar spinous process (L1-2 - L4-5) is in parallel, with wide contact area of apposing spinous processes. On the other hand, the spinous processes in L5 and S1 are not in parallel, due to the physiological lumbar lordosis and the difference of the each vertebral shape. For this reason, the contact area of L5 and S1 spinous process may be relatively smaller, resulting in less active inflammatory change with FDG uptake. In addition, as compared with data reported by Kwong et al., CT positivity was also higher in other locations, which is considered probably because of the difference of image quality of CT, i.e. low-dose CT in our study vs. diagnostic CT in Kwong's study.

In 27 patients who had serial scans, 35 (83\%) of 42 regions with positive FDG uptake at the first scan turned negative at the second scan. As expected, all 62 regions with positive CT findings at the first scan remained unchanged at the second scan. There were 17 regions in which PET was positive and CT was negative at the first scan, 
Lumbar spinous process FDG uptake

and PET turned negative at the second scan. In these 17 regions, degeneration on CT

was newly seen at the second scan only in four regions, suggesting that focal

accumulation around the LPS does not always result in degenerative change later.

There was no statistically significant difference in overall frequency of LSP

FDG uptake between males and females. However, comparing each age group, the peak of PET positivity in females appeared slightly earlier than that in males (Figure 2). This may be related to the higher frequency of age-related LSP degenerative changes in periand post-menopausal females [7], but the accurate reason remains unknown. Further investigations are required to address this issue.

Some limitations of this study include the quality of CT images, which was reduced to redundant radiation exposure. Additionally, evaluation by MRI was not performed and we did not compare PET images with MRI images. For this reason, we might not have evaluated soft tissue inflammatory change correctly. Although Baastrup disease causes low back pain [7-10,12-14,17], we did not evaluate patient symptoms in this retrospective study. Even if patients had back pain, it might be difficult to correlate the PET/CT finding with their symptoms, because many factors such as disk degeneration and facet osteoarthritis as well as Baastrup disease can cause back pain. Owing to the retrospective design, the range between the two scans in 27 patients varies. 
Lumbar spinous process FDG uptake

Unsettled period and any therapies might affect the FDG uptake, although we excluded patients who underwent chemotherapy or hormonal therapy during their follow-up period.

\section{Conclusions}

FDG uptake around the LSP was often seen in the mid-lumbar spine, regardless

of the patient's age. This uptake may represent a complex of degenerative changes in apposed spinous processes/inflammation of the interspinous process ligament/bursitis. As this commonly observed focal uptake corresponds to bone with or without some morphological changes, it should not be misjudged as bone metastasis. 
Lumbar spinous process FDG uptake

\section{References}

1. Boellaard R. Standards for PET image acquisition and quantitative data analysis. J

Nucl Med. 2009;50:11S-20S.

2. Zincirkeser S, Sahin E, Halac M, Sager S. Standardized uptake values of normal organs on 18F-fluorodeoxyglucose positron emission tomography and computed tomography imaging. J Int Med Res. 2007;35:231-6.

3. Rosen RS, Fayad L, Wahl RL. Increased ${ }^{18}$ F-FDG uptake in degenerative disease of the spine: Characterization with ${ }^{18}$ F-FDG PET/CT. J Nucl Med. 2006;47:1274-80.

4. Stumpe KD, Zanetti M, Weishaupt D, Hodler J, Boos N, Von Schulthess GK. FDG positron emission tomography for differentiation of degenerative and infectious endplate abnormalities in the lumbar spine detected on MR imaging. AJR Am J Roentgenol. 2002;179:1151-7

5. Yamashita H, Kubota K, Takahashi Y, Minamimoto R, Morooka M, Kaneko H, et al. Similarities and differences in fluorodeoxyglucose positron emission tomography/computed tomography findings in spondyloarthropathy, polymyalgia rheumatica and rheumatoid arthritis. Joint Bone Spine 2013;80:171-7

6. Baastrup CI. On the spinous processes of the lumbar vertebrae and the soft tissues between them, and on pathological changes in that region. Acta Radiol. 
Lumbar spinous process FDG uptake

$1933 ; 14: 52-5$.

7. Resnick D. Degenerative diseases of the vertebral column.

Radiology.1985;156:3-14.

8. Beks JW. Kissing spines: fact or fancy? Acta Neurochir. 1989;100:134-5.

9. Sartoris DJ, Resnick D, Tyson R, Haghighi P. Age-related alterations in the vertebral spinous processes and intervening soft tissues: radiologic-pathologic correlation. AJR Am J Roentgenol. 1985;145:1025-30.

10. Kwong Y, Rao N, Latief K. MDCT findings in Baastrup disease: disease or normal feature of the aging spine? AJR Am J Roentgenol. 2011;196:1156-9

11. Aylott CE, Puna R, Robertson PA, Walker C. Spinous process morphology: the effect of ageing through adulthood on spinous process size and relationship to sagittal alignment. Eur Spine J. 2012;21:1007-12.

12. Maes R, Morrison WB, Parker L, Schweitzer ME, Carrino JA. Lumbar interspinous bursitis (Baastrup disease) in a symptomatic population: prevalence on magnetic resonance imaging. Spine. 2008;33:E211-5.

13. Hui C, Cox I. Two unusual presentations of Baastrup's disease. Clin Radiol. 2007;62:495-7.

14. Jang EC, Song KS, Lee HJ, Kim JY, Yang JJ. Posterior epidural fibrotic mass 
Lumbar spinous process FDG uptake

associated with Baastrup's disease. Eur Spine J. 2010;19:S165-8.

15. Chen CK, Yeh L, Resnick D, Lai PH, Liang HL, Pan HB, et al. Intraspinal posterior epidural cysts associated with Baastrup's disease: report of 10 patients. AJR Am J Roentgenol. 2004;182:191-4.

16. Keorochana G, Taghavi CE, Tzeng ST, Lee KB, Liao JC, Yoo JH, et al. MRI classification of interspinous ligament degeneration of the lumbar spine: intraobserver and interobserver reliability and the frequency of disagreement. Eur Spine J. 2010;19:1740-5.

17. Ho L, Wassef H, Seto J, Henderson R. Multi-Level Lumbar Baastrup disease on F-18 FDG PET-CT. Clin Nucl Med. 2009;34:896-7.

18. Lin E. Baastrup's disease (kissing spine) demonstrated by FDG PET/CT. Skeletal Radiol. 2008;37:173-5.

19. Gorospe L, Jover R, Vicente-Bártulos A, González-Gordaliza C, de Llanos CG, García-Poza J. FDG-PET/CT demonstration of Baastrup disease ("Kissing" Spine). Clin Nucl Med. 2008;33:133-4.

20. Aliyev A, Saboury B, Kwee TC, Torigian DA, Basu S, Wulff Christensen H, et al. Age-related inflammatory changes in the spine as demonstrated by ${ }^{18}$ F-FDG-PET:observation and insight into degenerative spinal changes. Hell J Nucl 
Lumbar spinous process FDG uptake

Med. 2012;15:197-201. 
Lumbar spinous process FDG uptake

Figure Legends

Figure 1

A 60-year-old woman with a history of appendectomy for appendiceal follicular

lymphoma. Coronal images of CT (a), PET/CT fusion (b), PET (c), and sagittal images of CT (d), PET/CT fusion (e), PET (f) are demonstrated. Focal uptake is observed (SUVmax $=3.1$ ), corresponding to the lower portion (L3-4) of degenerated L3 and L4 spinous processes (arrows).

Figure 2: Frequency of PET positivity by age group.

PET positivity was found most frequently in the sixth decade. The peak of PET positivity in females appeared a little earlier than that in males. There was no statistically significant difference in overall frequency of FDG uptake between males and females.

Figure 3: Temporal changes of PET and CT findings between the first and the second scans. 
Lumbar spinous process FDG uptake

Thirty-five of 42 regions (83\%) with positive uptake turned negative at the second PET

scan, independent of CT findings, while all 62 regions with positive CT findings on the

first scan remained positive on the second scan.

$(+)$, positive; (-), negative 
a

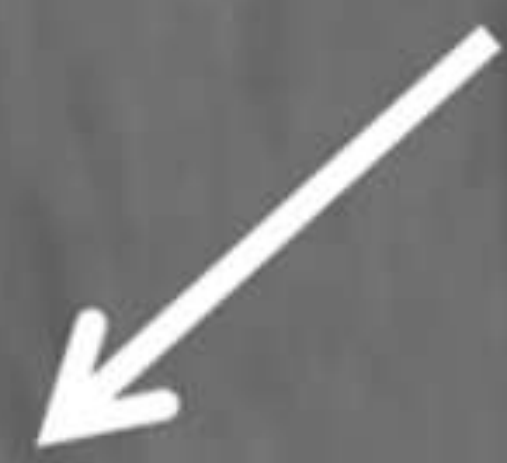




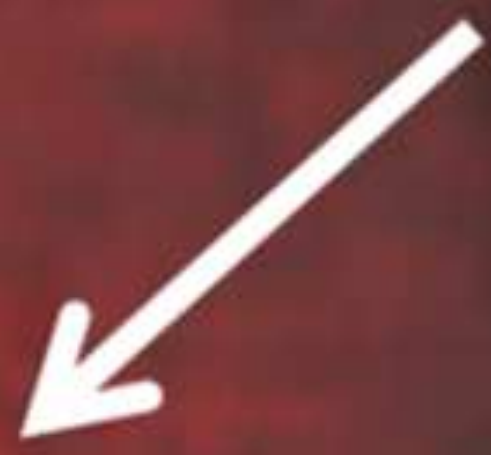




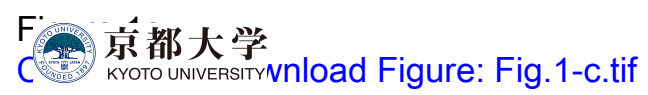

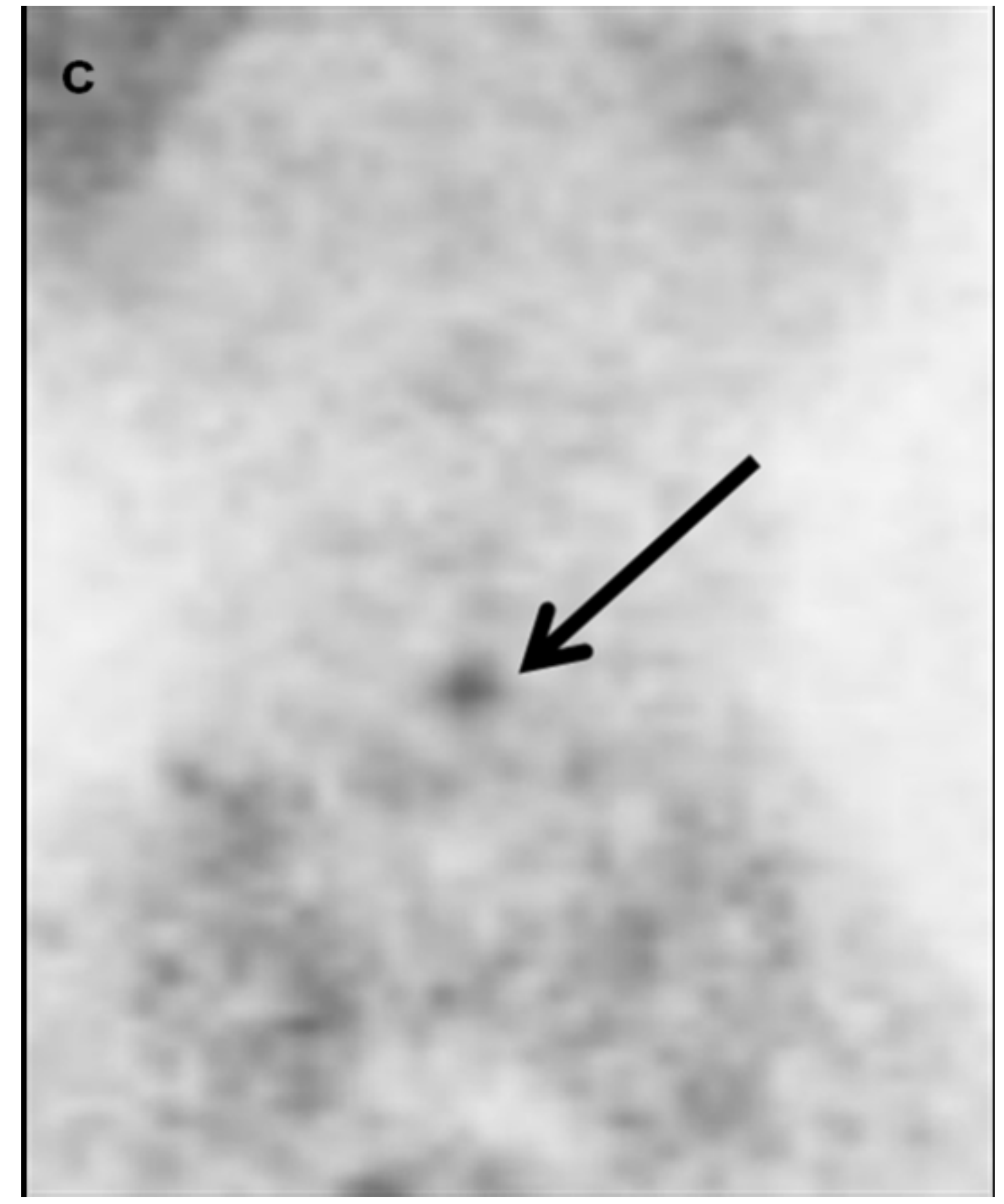




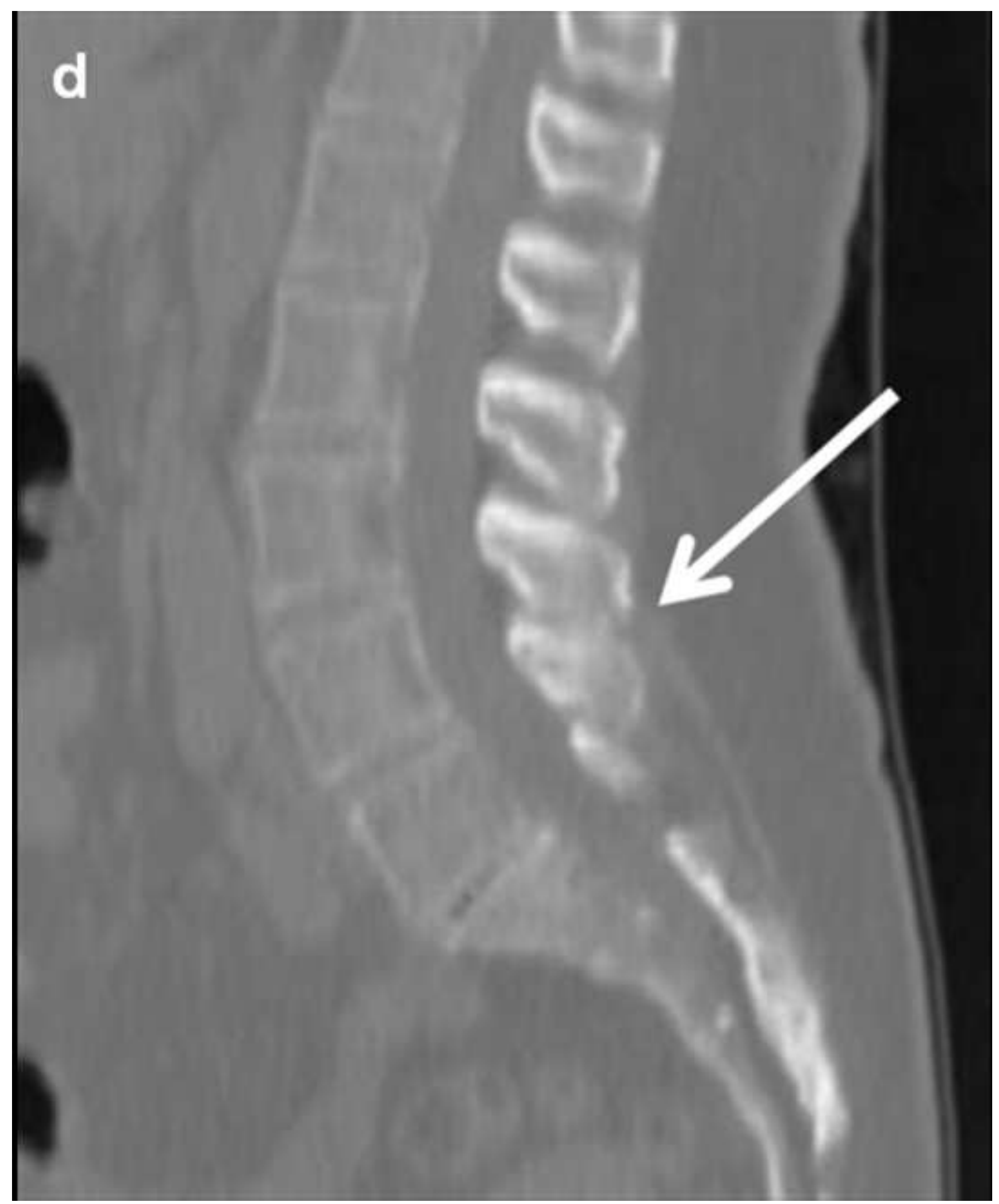




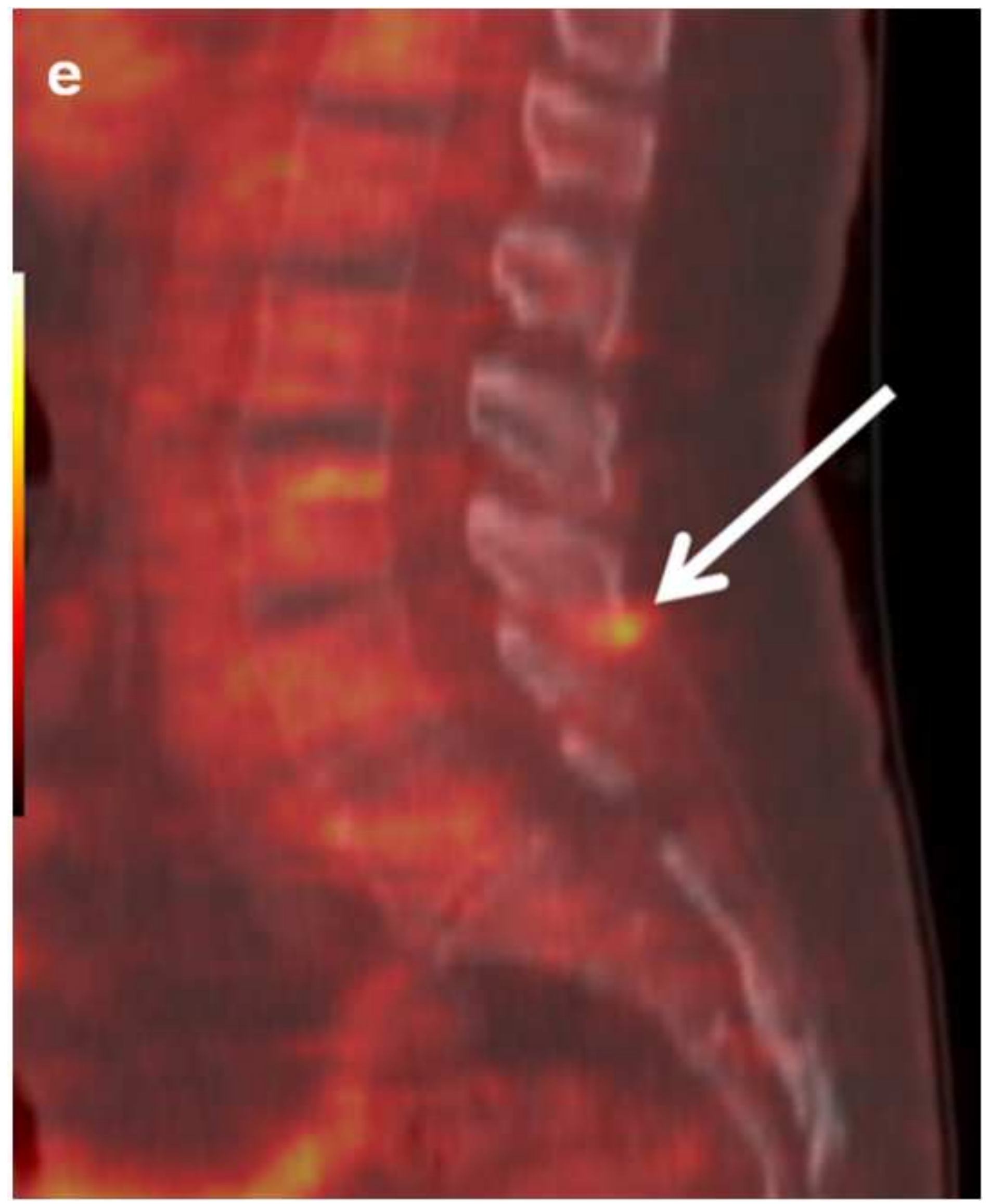




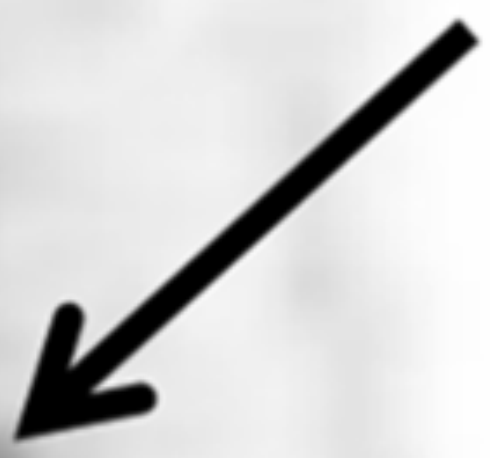




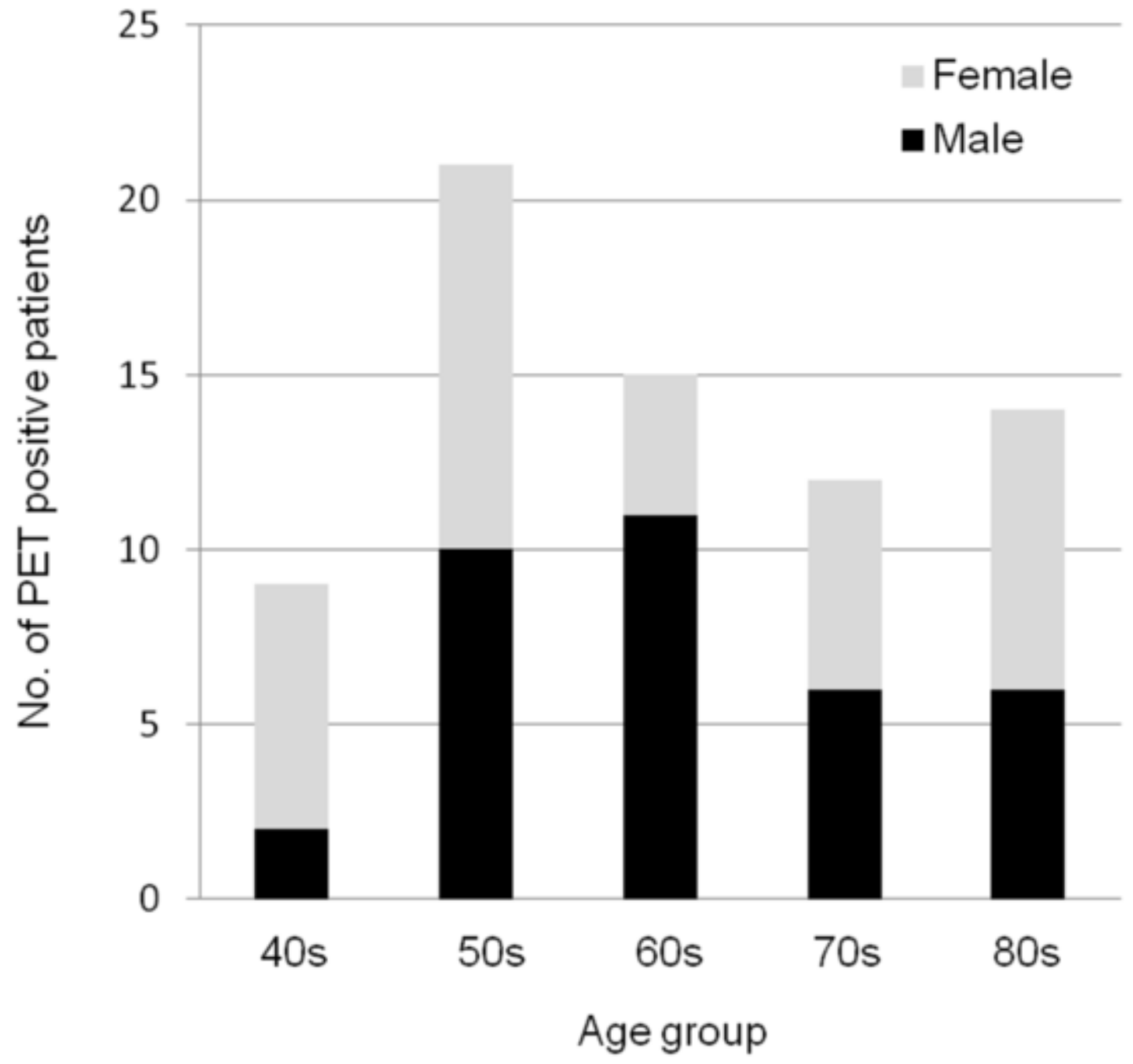



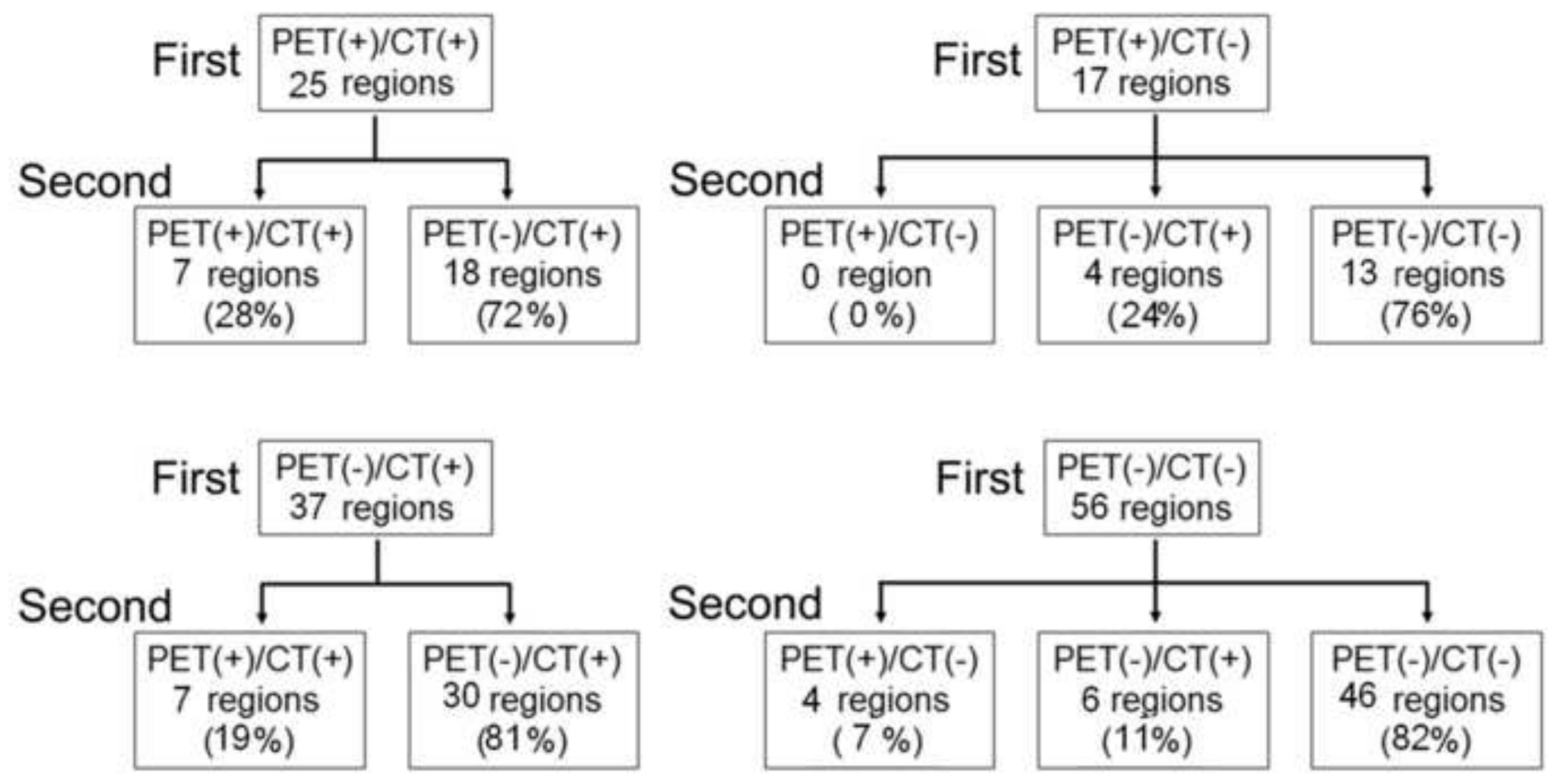
Table 1. Number of positive regions in each age group

\begin{tabular}{lcc} 
& & \\
\hline Age group & PET & CT \\
$40 s$ & $16(8 \%)$ & $58(29 \%)$ \\
$50 s$ & $30(15 \%)$ & $74(37 \%)$ \\
$60 \mathrm{~s}$ & $30(15 \%)$ & $108(54 \%)$ \\
$70 \mathrm{~s}$ & $20(10 \%)$ & $123(62 \%)$ \\
$80 \mathrm{~s}$ & $26(13 \%)$ & $144(72 \%)$ \\
\hline
\end{tabular}

Lumbar spinous FDG uptake 
Lumbar spinous FDG uptake

Table 2. Number of positive regions in each location

\begin{tabular}{ccc}
\hline Location & PET & CT \\
L1-2 & $19(10 \%)$ & $38(19 \%)$ \\
L2-3 & $22(11 \%)$ & $79(40 \%)$ \\
L3-4 & $39(20 \%)$ & $131(66 \%)$ \\
L4-5 & $35(18 \%)$ & $151(76 \%)$ \\
L5-S1 & $7(4 \%)$ & $108(54 \%)$ \\
\hline
\end{tabular}


Lumbar spinous FDG uptake

Table 3. Relationship between CT and PET findings for 1000 regions in 200 patients

\begin{tabular}{|c|c|c|c|c|}
\hline \multicolumn{2}{|c|}{ Total number of regions } & \multicolumn{2}{|c|}{ PET findings } & \multirow[t]{2}{*}{ SUVmax } \\
\hline & & Positive & Negative & \\
\hline CT positive & 507 & $72(14 \%)$ & $435(86 \%)$ & $1.19 \pm 0.32$ \\
\hline CT negative & 493 & $50(10 \%)$ & $443(90 \%)$ & $1.16 \pm 0.25$ \\
\hline
\end{tabular}

